\title{
An Intensity Consistent Approach to the Cross Sectional Analysis of Deformation Tensor Derived Maps of Brain Shape
}

\author{
C. Studholme, V. Cardenas, A. Maudsley, and M. Weiner \\ U.C.S.F., Dept of Radiology, VAMC (114Q), \\ 4150 Clement Street, San Francisco, \\ CA 94121, USA \\ cs1@itsa.ucsf .edu
}

\begin{abstract}
This paper describes a novel approach to the spatial filtering of deformation tensor derived maps of shape difference and shape change estimated in multi-subject studies of spatially normalised brain anatomy. We propose a spatial shape filter that combines tensor values locally which fall within regions of similar underlying intensity and therefore tissue. The filter additionally incorporates information derived from the spatial normalisation process to focus filtering more strongly within specific MRI intensities in regions where tissue intensities have been most consistently aligned in the spatial normalisation process. This is achieved using a statistical framework to introduce a measure of uncertainty of regional intensity correspondence. Results comparing the approach to conventional Gaussian filtering in the analysis of tensor derived measures of brain shape change in Alzheimer's disease and normal aging indicate significantly improved delineation of local atrophy patterns, particularly in cortical gray matter, without the need for explicit tissue segmentation.
\end{abstract}

\section{Introduction}

The use of non rigid registration transformations to capture spatial statistical measures of human anatomy is developing as a powerful tool in automated computational neuro anatomy 514 136941]. These methods make use of non rigid registration of brain MRI to a common anatomy, allowing the mapping of shape difference over the entire brain. Unlike ROI based techniques, such approaches are not limited to the quantification of specific anatomically defined structures, but can capture changes in all anatomical boundaries visible in the MRI which can be brought into correspondence. Once these spatially normalizing transformations have been estimated, it is possible to use a range of approaches to quantify shape difference from measurements derived from these transformations. One such powerful approach is that of tensor based morphometry which makes use of spatial derivatives of these transformations 53 . One of the challenges however in making use of this approach is how to handle the inherent spatial uncertainty in the underlying transformations, and resulting errors in the derivatives being analyzed. Spatial filtering of such fields can dramatically 


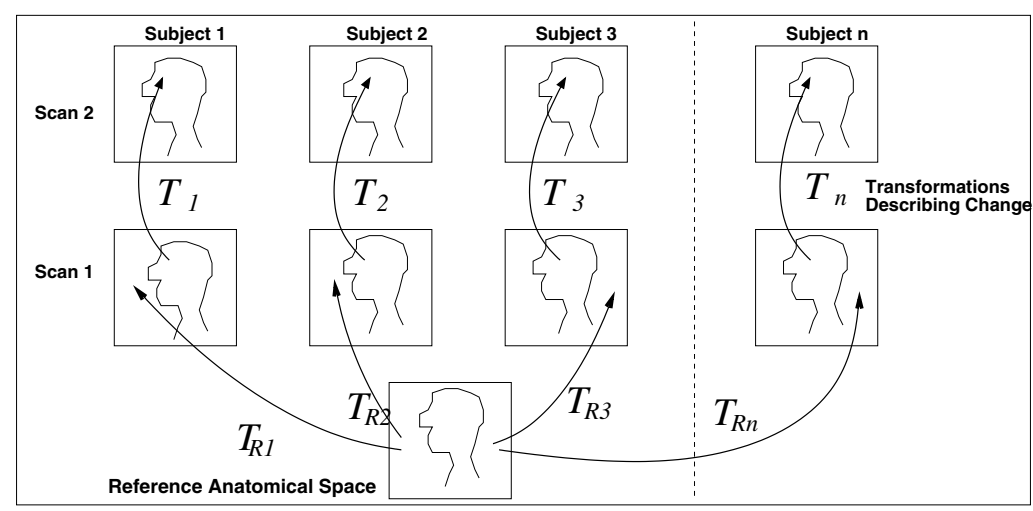

Fig. 1. Using non-rigid registration to capture local shape differences between subjects from the transformations $T_{R n}$, and shape changes within subject over time from the transformations $T_{\Delta n}$. To examine common patterns across subjects, maps of shape measures derived from these transformations may be evaluated and compared in the reference anatomy.

improve the statistical power of the approach, but at the expense of losing the fine-scale localization properties of these transformations. Filtering with uniform spatial filters is inherently sub-optimal because the underlying errors in registration estimates are themselves spatially varying: some locations are consistently aligned across all subjects, while other locations are poorly aligned because of underlying differences in anatomical topology. In this paper we explore a simple and novel approach to spatial filtering of these fields which incorporates information about the inherent spatial uncertainty in the spatial normalisation, while retaining the underlying anatomical localization accuracy provided by the alignment of visible boundaries in the images.

\section{Method}

\subsection{Tensor Morphometry: Spatial Maps of Shape from Registration}

The problem addressed in this paper is that of analyzing the spatial derivative fields used to describe shape difference (in purely cross sectional studies) and shape change (in serial or longitudinal MRI studies) over a group of individual anatomies. In such approaches non-rigid registration is used to capture shape difference between an individual and a reference anatomy or between repeat scans of an individual, as illustrated in figure 1. In such a study we have either imaged or synthesized a reference anatomy, producing a map of reference intensities $i_{R}\left(\mathbf{x}_{R}\right)$ over the points $\mathbf{x}_{R} \in X_{R}$ to use as a target for spatial normalisation. We then use this reference anatomical space to study a set $N$ of subject images with image intensities $\left\{i_{n}\left(\mathbf{x}_{n}\right), n \in N\right\}$ over points $\left\{\mathbf{x}_{n} \in X_{n}, n \in N\right\}$. In general we assume that the non-rigid registration transformation between the reference space and each subject $T_{R n}: \mathbf{x}_{R} \mapsto \mathbf{x}_{n}$ has been estimated by a registration procedure optimising an intensity similarity criteria, $C\left(i_{R}, i_{n}\right)$ between image intensities or 
alternatively extrapolating transformation models from aligned corresponding features.

In cross sectional tensor based morphometry $[5] 36] 10$, relative differences in shape between the reference and subject anatomies are described by the spatial derivatives of these transformations, evaluated at each point, to form maps of the local point-wise Jacobian,

$$
\mathbf{J}_{R n}\left(\mathbf{x}_{R}\right)=\frac{\partial T_{R n}\left(\mathbf{x}_{R}\right)}{\partial \mathbf{x}_{R}} .
$$

describing the relative local contractions or expansions of tissue from reference coordinates $\mathbf{x}_{R}$, to subject coordinates $T_{R n}\left(\mathbf{x}_{\mathbf{R}}\right)$. To remove directional information, the determinant of this matrix is often used as a summary measure of overall contraction or expansion at each point in the reference anatomy $J_{R n}(\mathbf{x})=\left|\mathbf{J}_{R n}(\mathbf{x})\right|$.

In serial MRI studies [11] a similar approach can be used to map local volumetric change between time points within a subject study using a non rigid transformation say $T_{n}$ between the time point 1 to 2 scans of subject $n$, which has itself been spatially normalised by a transformation $T_{R n}$. In the space of the common reference anatomy, these tissue contractions and expansions of an individual anatomy are described by the Jacobian mapping between time point 1 and time point 2 coordinates:

$$
\mathbf{J}_{n}\left(\mathbf{x}_{R}\right)=\frac{\partial T_{n}\left(T_{R n}\left(\mathbf{x}_{R}\right)\right)}{\partial T_{R n}\left(\mathbf{x}_{R}\right)}
$$

In this paper the approaches described to filtering local shape measures are applicable to both cross sectional and serial shape measures, and we will therefore refer to the general case of filtering shape measure $J_{n}$ at location $\mathbf{x}_{R}$.

\subsection{The Need for Spatial Filtering in Shape Analysis}

When estimating spatial transformations between anatomies, there are a number of fundamental reasons why there is remains an underlying ambiguity in the transformation estimates and the shape measures derived from them, which mean that post filtering of the shape fields is desirable. These include three main factors: Firstly that registration accuracy away from anatomical boundaries in the images is inherently under-determined and dependent on both the starting estimate and estimation methodology. Secondly: Geometric constraints on the allowable transformation estimates, such as transformation invertibility 2 and resolution of transformation parameterisation prevent a complete optimisation of $C\left(i_{R}, i_{n}\right)$ and therefore residual differences between $i_{R}(\mathbf{x})$ and $i_{n}\left(T_{R n}(\mathbf{x})\right)$ remain. Thirdly: the underlying variability of the location of disease induced effects across structures such as the cerebral cortex requires the study of their shape at both fine and coarse scales (i.e. a disease may remove local points of cortical gray matter distributed randomly within a given larger region such as the frontal lobe). 


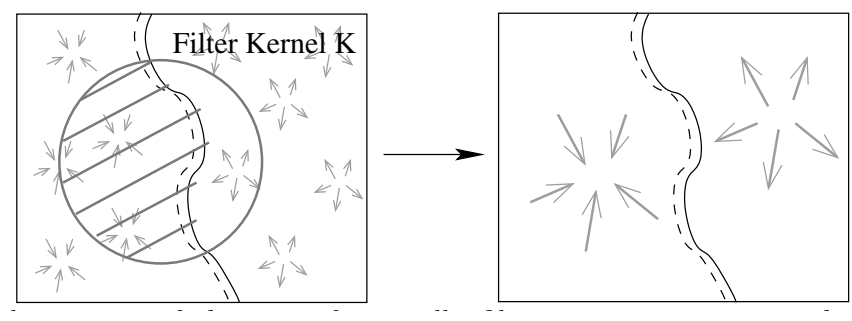

Fig. 2. An illustration of the aim of spatially filtering contractions and expansions in neighboring tissues separately so that anatomical boundaries are not lost.

\subsection{Spatial Filtering of Tensor Fields}

In the most direct approach to improving the signal to noise properties of the individual shape maps, we may consider applying a spatial filter to the Jacobian determinant map,

$$
\tilde{J}_{n}\left(\mathbf{x}_{R}\right)=\int_{\mathbf{k} \in K} J_{n}\left(\mathbf{x}_{R}-\mathbf{k}\right) \cdot f(\mathbf{k})
$$

to accumulate estimates of neighboring volume change into a more stable regional estimate, where $f(\mathbf{k})$ denotes the response of the spatial filter over neighborhood $\mathbf{k} \in K$. Previous approaches have used conventional Gaussian filters [6] which also usefully force the residual field toward a Gaussian random field for the purposes of statistical analysis. However, this does not take into account the fact that different tissues are expected to exhibit different shape properties. This is a particular problem when studying patterns of opposing local shape difference in fine scale structures such as the those in contracting cortical gray matter and expanding sulcal CSF in normal aging and degenerative disease. In this work we address this limitation by modifying the filtering of shape measures using an approach derived from that of the sigma filter [7]. Specifically, we propose a general probabilistic form of shape filter:

$$
\tilde{J}_{n}\left(\mathbf{x}_{R}\right)=\frac{1}{\Theta\left(\mathbf{x}_{R}\right)} \int_{\mathbf{k} \in K} J_{n}\left(\mathbf{x}_{R}-\mathbf{k}\right) \cdot f(\mathbf{k}) \cdot p_{x k}\left(\mathbf{x}_{R}, \mathbf{k}\right)
$$

where $p_{x k}\left(\mathbf{x}_{R}, \mathbf{k}\right)$ represents the statistical relationship between measurements at $\mathbf{x}_{R}$ and $\mathbf{x}_{R}-\mathbf{k}$ and,

$$
\Theta\left(\mathbf{x}_{R}\right)=\int_{\mathbf{k} \in K} f(\mathbf{k}) \cdot p_{x k}\left(\mathbf{x}_{R}, \mathbf{k}\right)
$$

normalizes for the volume under the local probabilistic neighborhood. The following section goes on the describe the design of a suitable formulation for $p_{x k}\left(\mathbf{x}_{R}, \mathbf{k}\right)$.

\subsection{A Measure of Association between Neighboring Shape Measurements}

One important aim is to use this filter operation to enforce the combination of shape measurements that fall in regions of similar underlying MRI intensity. 
However, since we are analyzing the maps in a common averaged anatomical space, we would ideally like each filtered subject map to conform to the anatomical structure in the averaged spatially normalised MRI of the entire group under study, rather than the spatially normalised individual MRI in which the measurements are made, since this may contain local tissue misalignment errors with respect to the common averaged MRI. We therefore choose to build a measure of association derived between the averaged intensity value,

$$
\mu^{\prime}\left(\mathbf{x}_{R}\right)=\frac{1}{N} \sum_{n \in N} i_{n}\left(\mathbf{T}_{R n}\left(\mathbf{x}_{R}\right)\right)
$$

of all spatially normalised MRI's, at the reference anatomy location $\mathbf{x}_{R}$ where the filter is being applied, and the spatially normalised individual MRI value,

$$
i_{n}^{\prime}\left(\mathbf{x}_{R}\right)=i_{n}\left(T_{R n}\left(\mathbf{x}_{R}\right)\right),
$$

at neighboring point $\left(\mathbf{x}_{R}-\mathbf{k}\right)$. In this work we examine the use of the conditional probability $p_{K}(i \mid \mu)$ as a regional filtering statistic. This is the probability of observing in region $K$, the spatially normalised individual MRI intensity $i$, given the averaged intensity of the group $\mu^{\prime}$ at the same location. We derive this by first constructing a local spatially weighted estimate of the joint probability distribution $p_{K}(i, \mu)$ between averaged MRI intensities and individual spatially normalised MRI intensities in the filter support around location $\mathbf{x}_{R}$. Algorithmically this is implemented as a discrete 2D histogram such that,

$$
p_{K}(i, \mu)=\frac{1}{\Gamma} \sum_{n \in N} \sum_{\mathbf{k} \in K} f(\mathbf{k}) \cdot \epsilon\left(i, i_{n}^{\prime}\left(\mathbf{x}_{R}-\mathbf{k}\right)\right) \cdot \epsilon\left(\mu, \mu^{\prime}\left(\mathbf{x}_{R}-\mathbf{k}\right)\right),
$$

where,

$$
\epsilon(\alpha, \beta)=\left\{\begin{array}{l}
1,\left\langle\frac{\alpha}{b}\right\rangle=\left\langle\frac{\beta}{b}\right\rangle \\
0, \text { otherwise }
\end{array}\right.
$$

counts equivalent intensities falling in bins with width $b,\langle\rangle$ indicates nearest integer, and the term

$$
\Gamma=\sum_{n \in N} \sum_{\mathbf{k} \in K} f(\mathbf{k})
$$

normalises by the weighted total number of voxels. This distribution captures the fraction of the filter neighborhood occupied by correctly aligned intensity pairs, together with the fraction of all combinations of mis-aligned intensity pairs. From this we then derive our intensity based statistical relationship between neighboring shape measurements, as the conditional probability $p_{K}(i \mid \mu)$ such that,

$$
p_{x k}\left(\mathbf{x}_{R}, \mathbf{k}\right)=p_{K}\left(i_{n}^{\prime}\left(\mathbf{x}_{R}-\mathbf{k}\right) \mid \mu^{\prime}\left(\mathbf{x}_{R}\right)\right)=\frac{p_{K}\left(i_{n}^{\prime}\left(\mathbf{x}_{R}-\mathbf{k}\right), \mu^{\prime}\left(\mathbf{x}_{R}\right)\right)}{p_{K}\left(\mu^{\prime}\left(\mathbf{x}_{R}\right)\right)}
$$

where $p_{K}\left(\mu^{\prime}\left(\mathbf{x}_{R}\right)\right)$ is the marginal probability of $\mu^{\prime}$. In regions where intensities are inconsistently aligned, $p_{K}(i \mid \mu)$ contains more than one peak and thus local shape measures lying in these peaks are accepted by the filter, reflecting the 
uncertainty in whether shape measures in these locations have been spatially normalised to the correct tissue. Conversely, in regions where MRI intensities are consistently brought into correspondence, $p_{K}(i \mid \mu)$ has one distinct peak for $\mu$ and forces the shape filter to accept only points falling in this narrow range.

\section{Results on Serial MRI Analysis in Alzheimer's Disease}

To illustrate the effect of using this proposed filtering on clinically derived shape maps, we have applied the filtering approach, using Gaussian spatial kernels for the filter $f()$, to the task of mapping brain shape change over time in a serial imaging study of dementia. 10 subjects diagnosed with Alzheimer's disease and 10 aging cognitively normal volunteers were imaged twice with an interval of between 1 and 3 years. A fine lattice entropy driven B-Spline deformation registration algorithm [11] was used to deform the later scan back to match the earlier scan, and spatially differentiated to produce a map of point-wise volume change in the coordinate system of the first time point scan. The transformation mapping between each first time point subject MRI scan and a single reference MRI was then evaluated using a multi-grid and multi-resolution registration, again driven by normalised mutual information [12. The individual maps of point-wise volume change between time points were then transformed to the coordinate system of the reference MRI. Global and local intensity inhomogeneity was removed from the intensity images and filters of three widths were then applied to each subject's atrophy map. A mean atrophy rate map was then calculated for the two groups. The results of comparing the three filter widths using conventional and intensity consistent filtering are shown in Figure 3 and enlarged for Alzheimer's in figure 4. As the filter width increases the ability of intensity consistent filter to retain fine structure in the cortex is clear. The overall level of atrophy is clearly greater in Alzheimer's over controls. The enlarged view shows the contrast detected in the atrophy rate between the white matter and gray matter ribbon over much of the cortex (note here that no explicit tissue segmentation has been applied).

\section{Discussion}

We have developed an approach to refining the analysis of deformation tensor fields that are derived from MRI scans of multiple subjects mapped to a common reference anatomy. It focuses filtering of local shape measures within regions of similar underlying MRI intensity and, additionally incorporates information about the local quality of the spatial normalisation of MRI intensities across the scans of subjects being studied. The filter does not require additional parameters to define its behaviour and automatically weights filtered shape measurements based on the local consistency in mapping structural image values at each point in the reference anatomy. One important factor to consider in this approach is that the filtered fields produced are fundamentally not continuous, but in fact reflect the underlying discontinuity in the anatomical structure being imaged. Statistically these fields therefore cannot be analysed using techniques based on assumptions such as those made in Gaussian random field theory. This is particularly important for correcting the statistical significance of any measurements 

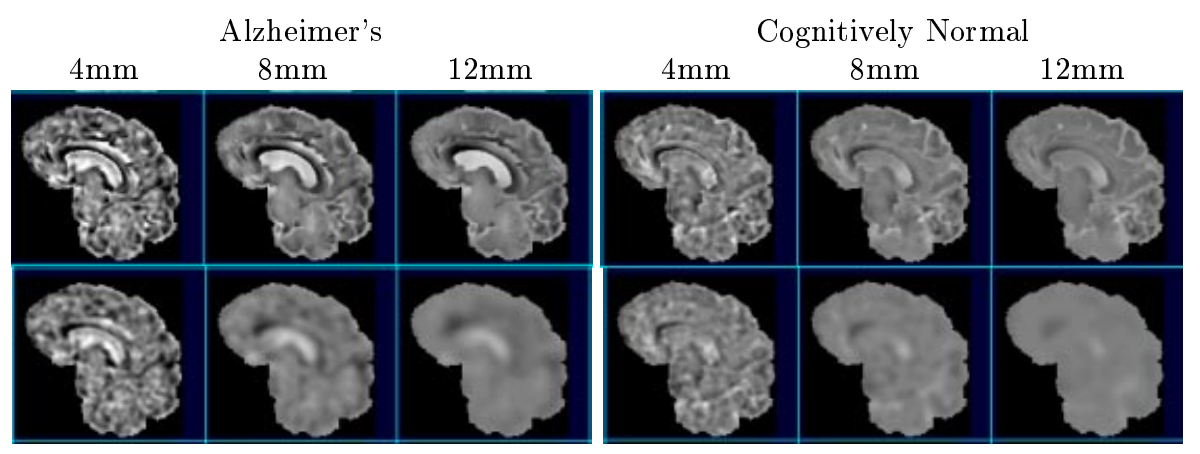

Fig. 3. Sagittal slices through reference anatomy showing the average spatially normalised maps of annualised volume change in 10 Alzheimer's patients (left) and 10 cognitively normal elderly subjects (right). Bottom row shows conventional spatially invariant Gaussian filtering, top row shows intensity consistent filtering. Averaged Jacobian determinant displayed as a gray scale thresholded at $-8 \%$ (black) and $+8 \%$ (white) per year.

for multiple comparisons. We are therefore developing approaches to analyzing statistical models of these fields based on permutation testing [ $]$. Although The approach is essentially free from spatial priors on the shape difference or change, the spatial filtering level employed is itself an implicit prior on any analysis. An interesting approach we are exploring is the multi-scale analysis possible in the non-Gaussian scale space of shape description produced by repeated application of tissue consistent spatial filters.

Acknowledgments. This work was primarily funded by the Whitaker Foundation. Image data used in this work was acquired as part of NIH grant P01 AG12435. The authors wish to thank Bruce Miller, Howard Rosen and Helena Chui for access to data and useful discussions on dementia and aging.

\section{References}

1. J. Ashburner, C. Hutton, R. Frackowiak, I. Johnsrude, C. Price, and K.J. Friston. Identifying global anatomical differences: Deformation-based morphometry. Neuroimage, 6:348-357, 1998.

2. G.E. Christensen and H.J. Johnson. Consistent image registration. Transactions on Medical Imaging, pages 568-582, 2001.

3. M. K. Chung, K. J. Worsley, T. Paus, C. Cherif, D. L. Collins, J. N. Giedd, J. L. Rapoport, and A. C. Evans. A unified statistical approach to deformation-based morphometry. Neuroimage, 14:596-606, 2001.

4. J.G. Csernansky, L. Wang, S. Joshi J.P. Miller M. Gado D. Kido D. McKeel, J.C. Morris, and MI. Miller. Early DAT is distinguished from aging by high-dimensional mapping of the hippocampus. Neurology, 55(11):1636-1643, 2000.

5. C. Davatzikos, M. Vaillant, S.M. Resnick, J.L. Prince, S. Letovsky, and R.N. Bryan. A computerised approach for morphological analysis of the corpus callosum. Journal of Computer Assisted Tomography, 20(1):88-97, 1996. 


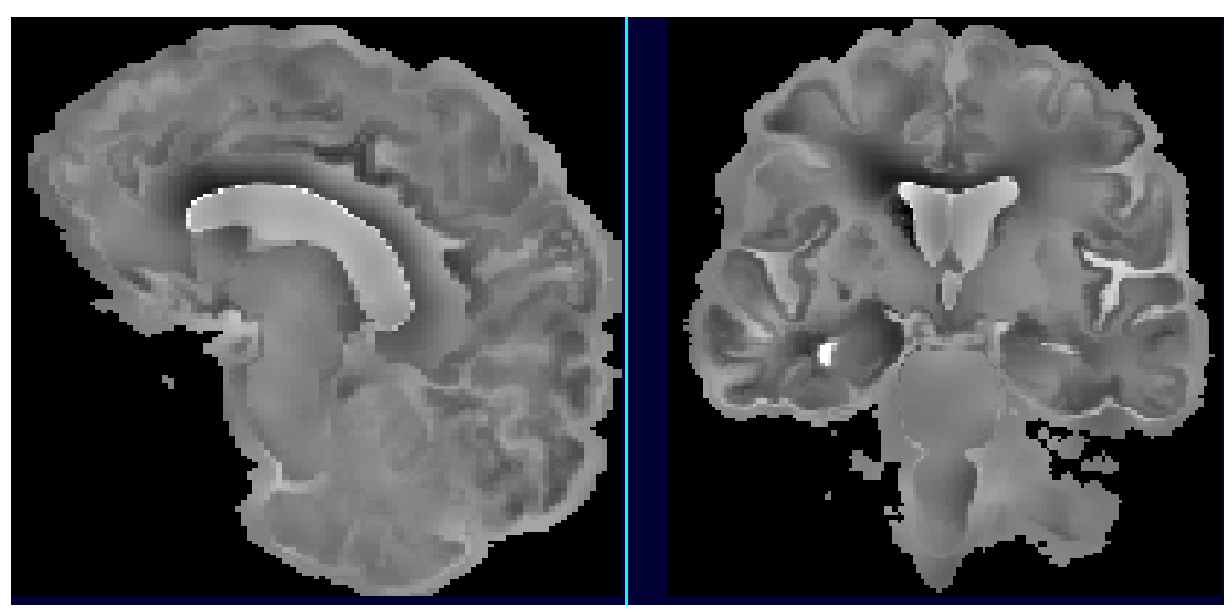

Fig. 4. Enlarged orthogonal views through the Alzheimer's average atrophy rate map, illustrating the different contraction rates visible in the cortical gray matter layer.

6. C. Gaser, H.P. Voltz, S. Kiebel, S. Riehemann, and H. Sauer. Detecting structural changes in whole brain based on nonlinear deformations- application to schizophrenia research. Neuroimage, 10:107-113, 1999.

7. J.S. Lee. Digital image smoothing and the sigma filter. Computer Vision, Graphics and Image Processing, 24(2):255-269, 1983.

8. T. E. Nichols and A. P. Holmes. Nonparametric permutation tests for functional neuroimagin: A primer with examples. Human Brain Mapping, 15:1-25, 2001.

9. D.J. Pettey and J.C. Gee. Using a linear diagnostic function and non-rigid registration to search for morphological differences between poluations: An example involving the male and female corpus callosum. In Proceedings SPIE Medical Imaging, volume 4322, page In Press, 2001.

10. C. Studholme, V. Cardenas, N. Schuff, H. Rosen, B. Miller, and M. Weiner. Detecting spatially consistent structural differences in alzheimer's and fronto temporal dementia using deformation morphometry. In Proceedings of 4 th International Conference on Medical Image Computing and Computer Assisted Interventions, 2001, pages 41-48, 2001.

11. C. Studholme, V. Cardenas, and M. Weiner. Building whole brain maps of atrophy from multi-subject longitudinal studies using free form deformations. In Proceedings of ISMRM 2001, Glasgow, Scotland, 2001.

12. C. Studholme, V. Cardenas, and M. Weiner. Multi scale image and multi scale deformation of brain anatomy for building average brain atlases. In Proceedings SPIE Medical Imaging, volume 4322-60, pages 557-568, 2001.

13. J.P. Thirion and G. Calmon. Deformation analysis to detect and quantify active lesions in three-dimensional medical image sequences. IEEE Transactions on Medical Imaging, 18(5):429-441, 1999.

14. P. Thompson, R. Woods, M. Mega, and A. Toga. Mathematical/Computational challenges in creating deformable and probabilistic atlases of the human brain. Human Brain Mapping, 9:81-92, 2000. 